BMJ Surgery, Interventions, \& Health Technologies

\title{
1-year cost-utility analysis of prostate artery embolization (PAE) versus transurethral resection of the prostate (TURP) in benign prostatic hyperplasia (BPH)
}

Nikisha Patel (D) , ${ }^{1}$ Nathan Yung (D) ,, Ganesh Vigneswaran, ${ }^{2}$ Laure de Preux, ${ }^{3}$ Drew Maclean, ${ }^{2}$ Mark Harris, ${ }^{4}$ Bhaskar Somani (D) , ${ }^{4}$ Timothy Bryant, ${ }^{2}$ Nigel Hacking, ${ }^{2}$ Sachin Modi (1) ${ }^{2}$

\section{ABSTRACT}

Objective To determine whether prostate artery embolization (PAE) is a cost-effective alternative to transurethral resection of the prostate (TURP) in the management of benign prostate hyperplasia (BPH) after 1-year follow-up.

Design, setting and main outcome measures $A$ retrospective cost-utility analysis over a 12-month time period was conducted to compare the two interventions from a National Health Service perspective. Effectiveness was measured as quality-adjusted life years (QALYs) derived from data collected during the observational UK Register of Prostate Embolisation (UK-ROPE) Study. Costs for both PAE and TURP were derived from University Hospital Southampton, a tertiary referral centre for BPH and the largest contributor to the UK-ROPE. An incremental cost-effectiveness ratio (ICER) was derived from cost and QALY values associated with both interventions to assess the cost-effectiveness of PAE versus TURP. Further sensitivity analyses involved a decision tree model to account for the impact of patient-reported complications on the cost-effectiveness of the interventions.

Results The mean patient age for TURP ( $n=31)$ and PAE $(n=133)$ was 69 and 65.6 years, respectively. In comparison to TURP, PAE was cheaper due to shorter patient stays and the lack of necessity for an operating theatre. Analysis revealed an ICER of $£ 64798.10$ saved per QALY lost when comparing PAE to TURP after 1-year follow-up.

Conclusion Our findings suggest that PAE is initially a cost-effective alternative to TURP for the management of $\mathrm{BPH}$ after 1-year follow-up. Due to a higher reintervention rate in the PAE group, this benefit may be lost in subsequent years.

Trial registration number NCT02434575.

\section{INTRODUCTION}

Prostate artery embolization (PAE) is now considered a standard treatment option for the management of lower urinary tract symptoms (LUTS) secondary to benign

\section{Significance of study}

What is already known about this subject?

- This is the first UK-based cost comparison of two procedures commonly used in the treatment of a common condition affecting a large number of men (BPH). Prostate artery embolization (PAE) has gained evidence over the last 3 years and following the UK-ROPE Study, that has been approved by National Institute for Health and Care Excellence for use in the UK. Transurethral resection of the prostate (TURP) is the current gold standard technique for treating $\mathrm{BPH}$.

What are the new findings?

- The findings suggest that PAE is more cost-effective than TURP at 1-year follow-up.

\section{How might these results affect future} research or surgical practice?

- This may lead to greater uptake of PAE in the UK. Not all centres offer this service. This study may lead to a reimbursement code for PAE which may help some trusts offer this service. Many men would prefer to have PAE due to the lower risks associated and that it is performed under local anesthesia as a day case procedure.

prostatic hyperplasia (BPH) in the UK since the updated National Institute for Health and Care Excellence (NICE) guidance in 2018. ${ }^{1}$ In Europe, PAE is included in the European Association of Urology guidelines as a standard procedure, but is not supported in the American Urological Association guidelines, which recommends it use in clinical trials only. There have been multiple studies that have demonstrated the clinical effectiveness of PAE versus transurethral resection of the prostate (TURP), with significant improvements in symptoms, as measured by the 
International Prostatic Symptom Score (IPSS) reduction. ${ }^{23}$ However, there are limited data on the cost utility of TURP compared with PAE.

The prevalence of BPH is known to increase with age. ${ }^{4}$ This, combined with the UK ageing population, suggests that management of BPH-related LUTS imposes a growing financial burden on the UK healthcare system. ${ }^{5}$ The limited budget of the National Health Service (NHS) requires efficient resource allocation to ensure maintenance of high-quality patient care. A recent American cost analysis suggested that TURP is more expensive than PAE, but recognized that a more comprehensive cost analysis, such as the cost of physician time and cost of complications, was required to improve accuracy. ${ }^{6}$ Currently, no UK-based cost-utility analysis has been published.

TURP has good clinical outcomes, low mortality rates $(\sim 0.1 \%)^{7}$ and remains the gold standard surgical treatment for BPH-related LUTS. ${ }^{8}$ However, morbidity such as postoperative retrograde ejaculation and requirement for potential hospitalization and catheterization can reduce its cost-effectiveness. ${ }^{8}$ Monopolar transurethral vaporization of the prostate (TUVP) and holmium laser enucleation of the prostate (HoLEP) remain costeffective alternative surgical options. A systematic review comparing cost utility of surgical treatments concluded that TUVP was cheaper but less effective than TURP, whereas HoLEP was more cost-effective than TURP. ${ }^{9}$ Despite the benefits of HoLEP, the greatest obstacles to its widespread implementation lie with the high start-up cost, technical difficulties and learning curve associated with the procedure. ${ }^{10}$

PAE offers an alternative non-surgical, minimally invasive option ${ }^{11}$ and data suggest that the cost of PAE may be lower than TURP, while maintaining a lower morbidity rate. ${ }^{12}$ The aim of this study was to carry out a retrospective cost-utility analysis comparing TURP and PAE for the treatment of BPH-related LUTS using the UK Register of Prostate Embolisation (UK-ROPE) Study published in $2018 .^{13}$

The UK-ROPE is a national observational database of patients treated with PAE or surgical procedures from 20 centres, held by the Cardiff-based independent research organization 'Cedar', funded by NICE. Procedure costs were covered either through local commissioning streams or through a grant from Cook Medical (Europe), where this was not possible. Male patients were enrolled for either PAE, TURP, open prostatectomy or HoLEP from July 2014 to January 2016. All patients had consultation with a urologist and interventional radiologist prior to PAE. Outcome data including IPSS, quality of life (QoL) and International Index of Erectile Function (IIEF) were collated from questionnaires. Patients were followed up at 1 month, 3 months, 6 months and 12 months post procedure. Various clinician-reported complications were recorded initially post procedure, while patient-reported complications (hematuria, hematospermia, incontinence, urinary infection and retrograde ejaculation) were identified from questionnaire responses at each time point. Briefly, the UK-ROPE demonstrated that PAE showed a statistically significant improvement in IPSS and QoL post procedure, but the improvement in outcomes following TURP was greater. The registry did not have any stipulations on operator/centre experience for either TURP or PAE to simulate 'real-world' practice. Due to the experimental nature of PAE at the time of the study, all centres were proctored by an experienced operator for at least six cases. Due to the established practice of TURP in the UK, no proctoring was performed for cases.

\section{METHODS}

Sample

The UK-ROPE Study collected 1-month, 3-month, 6-month and 12-month IPSS values from 133 patients who underwent PAE and 31 patients who underwent TURP. There was no requirement for similar sample sizes in registry design which resulted in different cohort sizes. This study focuses on outcomes observed at 12 months post intervention and includes all registry patients with IPSS pre procedure and at 12-month follow-up.

For specific details regarding the selection process of participants, please refer to the original UK-ROPE Study. ${ }^{13}$ Selection of treatment (PAE or TURP) was left to patient and operator discretion. In the original UK-ROPE Study, there were no significant differences in baseline IPSS, QOL, IIEF, prostate volume or maximum urinary flow rate. Patients were marginally younger in the PAE group (median 67 vs 70 years, Mann-Whitney $\mathrm{U}$ p=0.001).

\section{Analytic horizon and choice of analysis}

A 2019 meta-analysis compared PAE and TURP by assessing four studies, all of which had a follow-up time period of at least 12 months. ${ }^{2}$ This study therefore also used a 12-month time horizon where the outcomes of PAE and TURP were observed at 12 months post intervention. This is in line with the original UK-ROPE Study which also aimed to assess outcomes at 12 months.

BPH-related LUTS affect QoL, ${ }^{14}$ hence a cost-utility analysis was performed to compare TURP and PAE in relation to utility. The study period being 12 months, the utility values can be expressed as quality-adjusted life years (QALYs). The 12 month IPSS values of each patient collected during the UK-ROPE Study were used as the basis of this cost-utility analysis, where they were converted into QALY values and used along with the cost of interventions to calculate an incremental cost-effectiveness ratio (ICER) expressed in units of cost per QALY. For this analysis, the breakdown score of the IPSS was evaluated.

\section{Cost of interventions}

A reference year of 2016 was applied with regard to costs. The 2016 costs of both PAE and TURP procedures (table 1) were obtained from University Hospital Southampton (UHS). This site is a tertiary referral centre for BPH and routinely provides TURP for patients and was the largest single contributor to the UK-ROPE Study. 
Table 12016 costs of interventions (in £ per patient) carried out in the UK-ROPE Study

\begin{tabular}{|c|c|}
\hline Intervention & $\begin{array}{l}\text { Cost (£ per } \\
\text { patient) }\end{array}$ \\
\hline \multicolumn{2}{|l|}{ PAE (UHS) } \\
\hline $\begin{array}{l}\text { Interventional radiology (IR) clinic (pre and } \\
\text { post) }\end{array}$ & 100 \\
\hline CT angiography & 250 \\
\hline $\begin{array}{l}\text { Procedural and postprocedural staffing costs } \\
\text { (IR room time, scrub team, recovery and } \\
\text { radiographer) }\end{array}$ & 500 \\
\hline Sheath, wire, catheter and microcatheter & 300 \\
\hline Embolization pack (drapes and consumables) & 50 \\
\hline Urology clinic (pre and post) & 100 \\
\hline Urodynamics (optional) & 200 \\
\hline MRI (optional) & 350 \\
\hline Ultrasound scan (optional) & 150 \\
\hline Total & 2000 \\
\hline \multicolumn{2}{|l|}{ TURP (UHS) } \\
\hline Theatre time & 1126 \\
\hline Ward costs & 595 \\
\hline Anesthetist cost & 264 \\
\hline Surgeon cost & 201 \\
\hline Materials & 180 \\
\hline Pathology & 162 \\
\hline Miscellaneous & 400 \\
\hline Clinic (pre and post) & 100 \\
\hline Total & 3028 \\
\hline \multicolumn{2}{|c|}{ TURP (National Schedule of Reference Costs 2015/2016)*15 } \\
\hline $\begin{array}{l}\text { Transurethral prostate resection procedures } \\
\text { with CC Score } 6+\end{array}$ & 4359.52 \\
\hline $\begin{array}{l}\text { Transurethral prostate resection procedures } \\
\text { with CC Score } 3-5\end{array}$ & 2992.66 \\
\hline $\begin{array}{l}\text { Transurethral prostate resection procedures } \\
\text { with CC Score } 0-2\end{array}$ & 2537.97 \\
\hline
\end{tabular}

*Document published by the Department of Health and Social Care which outlines spending across 237 National Health Service providers for delivering healthcare to patients in 2015 and 2016

CC, complications and comorbidities; PAE, prostate artery embolization; TURP, transurethral resection of the prostate; UHS, University Hospital Southampton.

At this site, more than 350 PAE procedures have been performed and in depth costs of all aspects relating to PAE were obtained. Due to the novel nature of this treatment, no tariff code is yet publicly available for PAE. In order to account for any local variability, the cost of TURP was obtained from UHS, which was in accordance with the National Schedule of Reference Costs 2015/2016 ${ }^{15}$ published by the UK Department of Health and Social Care. Assumptions made in the cost estimations include a day case procedure for PAE (vs overnight stay for TURP, supported by median length of stay values in the
Table 2 Lower urinary tract symptoms that comprised the International Prostate Symptom Score

\begin{tabular}{ll}
\hline Symptom type & Symptom \\
\hline Obstructive & Incomplete emptying \\
& Intermittency \\
& Straining \\
& Weak stream \\
Irritative & Urgency \\
& Frequency \\
& Nocturia \\
\hline
\end{tabular}

UK-ROPE Registry). PAE is performed under local anesthetic compared with general anesthetic for TURP. Procedural staffing costs are notably lower for PAE due to the absence of an anesthetist/operating department practitioner and lower interventional radiology room staffing numbers. Reference was also made against other UK trusts in the UK-ROPE Registry to ensure similar cost estimation of interventions. Post-PAE urodynamics, MRI and ultrasound are only recommended in a research/early practice setting (conducted in some UK-ROPE centres at the time of the registry), whereas these investigations are no longer considered standard practice and are therefore deemed optional costs. 'Miscellaneous' in the TURP costs are due to a combination of recovery cost, junior medical staffing, pharmacy and therapy.

\section{IPSS and QALY}

The IPSS comprises seven BPH-related LUTS, which can be categorized into obstructive and irritative symptoms (table 2). Patients in the UK-ROPE Study were asked to assign a score from 0 to 5 to indicate how often they experienced each symptom ( 0 being not at all and 5 being almost always). The individual scores were then added up to give a total IPSS out of 35 .

For the purpose of performing a cost-utility analysis, the 12-month IPSS values of the patients included in the UK-ROPE Study were converted into QALYs via a method suggested by Kok et al, ${ }^{16}$ which involved equating IPSS to a utility value dependent on scores for obstructive and irritative symptoms. Each patient's obstructive symptoms score and irritative symptoms score were equated to a level representing the severity of obstructive and irritative symptoms, which was associated with a corresponding utility value. The method of conversion of IPSS to utility values and the subsequent individual QALY values derived from each patient's IPSS scores are available as supporting information (online supplemental tables S1 and S2).

\section{Sensitivity analysis}

Complications of interventions

The UK-ROPE Study used questionnaires at 1 month, 3 months, 6 months and 12 months post intervention to identify patient-reported complications (hematuria, 
hematospermia, incontinence, urinary infection and retrograde ejaculation). Patients were asked to indicate all complications they experienced since the intervention. In order to provide a comprehensive evaluation of PAE and TURP, the cost-utility analysis took into account both the costs and QALYs associated with the patientreported complications at 12 months post intervention.

\section{Cost of complications}

The costs assigned to the patient-reported complications considered in this analysis are available as supporting information (online supplemental table S3). Hematuria and hematospermia were assigned a cost of nil, as current evidence suggests that these complications tend to be benign and self-limiting. ${ }^{17} 18$ This was in accordance with data recorded in the UK-ROPE which highlighted that of all the patients which reported hematuria (76), only $3.95 \%$ of patients required readmission for assessment of hematuria, with the remainder selfresolved with 4 weeks post procedure. ${ }^{13}$ Of the patients reassessed, none required intervention for their hematuria-as such, no treatment is usually indicated. No patients required further assessment or treatment for hematospermia.

While hematuria requiring intervention is a rare, clot retention can occur, which usually requires inpatient admission, resulting in a notable cost. In order to account for this, further analysis was completed whereby a cost based on the 2015/2016 NHS Reference $\operatorname{costs}^{19}$ is assigned to hematuria in sensitivity analysis 3 .

Previous studies have shown that BPH cases increase linearly with age, with incidence peaking between 75 and $79 .^{20}$ While treatments for retrograde ejaculation are available, the complication was assigned a cost of nil, as the main indication to resolve the complication is to achieve pregnancy through antegrade ejaculation, which would be unlikely in this cohort of patients. ${ }^{21}$

The costs associated with managing incontinence were based on the 2015/2016 NHS reference costs. ${ }^{19}$ The total cost of managing urinary infection was derived from the cost of performing urinalysis ${ }^{22}$ and antibiotics (which were based on the 2015/2016 NHS Prescription Services Drug Tariff). ${ }^{23}$ A full breakdown of the costs of complications is available as supporting information (online supplemental table 4).

\section{Modeling and decision tree}

For each intervention, patients were grouped according to the combination of patient-reported complications they experienced within 12 months post intervention, giving rise to a series of outcomes summarized in table 3 .

The individual complications of each patient are available to view as supporting information (online supplemental table S2)). For each outcome, the QALY values derived from individual patients' IPSS values were used to calculate an average QALY value. The probability of each outcome was also derived.

\section{Sensitivity analysis 1}

Figure 1 shows a decision tree that split into two arms, one for each intervention. Each arm consisted of the different outcomes and their associated probabilities, total costs and assigned QALY values (table 3). In each arm, the total cost of an outcome was derived from the sum of the intervention cost and cost of managing the complications observed. The individual QALY value of each patient-reported complication was considered for each branch to account for its impact on QoL post intervention in the analysis.

Outcomes where urinary infection was present were assigned a QALY value of 0.93 , while outcomes where incontinence was present were assigned a QALY value of 0.89 , in line with the literature. ${ }^{24}$

The average QALY value for the group of patients who experienced no complications was 0.96 in the PAE arm and 0.99 in the TURP arm (outcome 11 and 7 in table 3, respectively). For the purpose of a sensitivity analysis, where urinary infection and/or incontinence was absent, but other complications (hematuria, hematospermia and/or retrograde ejaculation) were present, a QALY value of 0.96 was assigned in the PAE arm and 0.99 in the TURP arm. This is because these complications had no pre-existing associated utility values in the literature and can be considered painless/benign. Therefore, such complications were assumed to be associated with 'perfect health'.

The QALY values and total costs associated with each outcome were used with corresponding probability values, to derive an expected cost and expected QALY for each intervention. An ICER was subsequently derived.

\section{Sensitivity analysis 2}

Figure 2 shows the decision tree of a further sensitivity analysis that involved assigning all outcomes associated with complications a QALY of 0.89 , as this was the lowest QALY associated with a patient-reported complication (incontinence). This allowed another ICER to be derived to investigate the impact of complications on the costeffectiveness of PAE versus TURP, assuming the complications were associated with worse QALY values.

\section{Sensitivity analysis 3}

To account for the potential complication of significant hematuria requiring intervention, a further analysis is conducted as an extension of sensitivity analysis 2. Using the same QALY values used in sensitivity analysis 2, all patients experiencing hematuria are assigned a cost of $£ 1316.50$ in accordance to the $2015 / 2016$ NHS National Tariff. Although it is unlikely that the majority of patients experiencing hematuria will accrue such a significant cost, this allows a conservative ICER to be derived to investigate the impact of significant hematuria on the cost-effectiveness of PAE versus TURP. 
Table 3 Summary of complications, number of patients, probabilities, QALY values and total costs (in £ per patient) associated with outcomes for PAE and TURP 12 months post intervention

\begin{tabular}{|c|c|c|c|c|c|c|c|}
\hline Outcome & Complications & $\begin{array}{l}\text { Total cost }(£ \\
\text { per patient) }\end{array}$ & $\begin{array}{l}\text { Number of } \\
\text { patients }\end{array}$ & Probability & $\begin{array}{l}\text { Average } \\
\text { QALY } \pm \text { SD }\end{array}$ & $\begin{array}{l}\text { Assigned QALY } \\
\text { for sensitivity } \\
\text { analysis } 1\end{array}$ & $\begin{array}{l}\text { Assigned QALY } \\
\text { for sensitivity } \\
\text { analyses } 2 \text { and } 3\end{array}$ \\
\hline \multicolumn{8}{|l|}{ PAE } \\
\hline 1 & HU, HS, UI & 2010.04 & 1 & 0.008 & $1.00 \pm 0.00$ & 0.93 & 0.89 \\
\hline 2 & HU, HS, RE & 2000.00 & 4 & 0.030 & $0.97 \pm 0.02$ & 0.96 & 0.89 \\
\hline 3 & $\mathrm{HU}, \mathrm{HS}$ & 2000.00 & 6 & 0.045 & $0.95 \pm 0.03$ & 0.96 & 0.89 \\
\hline 4 & $\mathrm{HU}, \mathrm{RE}$ & 2000.00 & 3 & 0.023 & $0.97 \pm 0.03$ & 0.96 & 0.89 \\
\hline 5 & $\mathrm{HU}$ & 2000.00 & 16 & 0.120 & $0.97 \pm 0.03$ & 0.96 & 0.89 \\
\hline 6 & HS, RE & 2000.00 & & 0.008 & $0.97 \pm 0.00$ & 0.96 & 0.89 \\
\hline 7 & HS & 2000.00 & 5 & 0.038 & $0.97 \pm 0.03$ & 0.96 & 0.89 \\
\hline 8 & I & 3203.33 & & 0.008 & $0.97 \pm 0.00$ & 0.89 & 0.89 \\
\hline 9 & UI & 2010.04 & 5 & 0.038 & $0.93 \pm 0.05$ & 0.93 & 0.89 \\
\hline 10 & RE & 2000.00 & 6 & 0.045 & $0.97 \pm 0.05$ & 0.96 & 0.89 \\
\hline 11 & Nil & 2000.00 & 85 & 0.639 & $0.96 \pm 0.03$ & 0.96 & 0.96 \\
\hline Total & - & - & 133 & 1.000 & $0.96 \pm 0.03$ & - & - \\
\hline \multicolumn{8}{|l|}{ TURP } \\
\hline 1 & $\mathrm{HU}, \mathrm{HS}$ & 3028.00 & 1 & 0.032 & $1.00 \pm 0.00$ & 0.99 & 0.89 \\
\hline 2 & $\mathrm{HU}, \mathrm{I}, \mathrm{RE}$ & 4231.33 & 1 & 0.032 & $0.94 \pm 0.00$ & 0.89 & 0.89 \\
\hline 3 & HU, UI, RE & 3038.04 & 1 & 0.032 & $1.00 \pm 0.00$ & 0.93 & 0.89 \\
\hline 4 & $\mathrm{HU}, \mathrm{RE}$ & 3028.00 & 11 & 0.355 & $0.98 \pm 0.03$ & 0.99 & 0.89 \\
\hline 5 & $\mathrm{HU}$ & 3028.00 & 7 & 0.226 & $0.97 \pm 0.04$ & 0.99 & 0.89 \\
\hline 6 & $\mathrm{RE}$ & 3028.00 & 5 & 0.161 & $0.98 \pm 0.02$ & 0.99 & 0.89 \\
\hline 7 & Nil & 3028.00 & 5 & 0.161 & $0.99 \pm 0.02$ & 0.99 & 0.99 \\
\hline Total & & - & 31 & 1.000 & $0.98 \pm 0.03$ & - & - \\
\hline
\end{tabular}

${ }^{*}$ Total cost (in $£$ per patient) for each outcome was derived by adding the intervention cost (see table 1) to the cost of managing the observed complications (see online supplemental table S3).

HS, hematospermia; HU, hematuria; I, incontinence; PAE, prostate artery embolization; QALY, quality-adjusted life year; RE, retrograde ejaculation; TURP, transurethral resection of the prostate; UI, urinary infection.

\section{RESULTS}

A total of 133 patients who underwent PAE and 31 patients who underwent TURP were analyzed. The mean age of PAE patients was 65.6 years $(\mathrm{SD}=6.7)$. The mean age of TURP patients was 69 years $(\mathrm{SD}=7.4)$. The average QALY values of the $133 \mathrm{PAE}$ patients and 31 TURP patients were $0.96(\mathrm{SD}=0.03)$ and $0.98(\mathrm{SD}=0.03)$, respectively. The average cost per patient of PAE and TURP was $£ 2000$ and $£ 3028$, respectively. This generated an ICER of $£ 64798.10$ saved per QALY lost. Within this cohort, 36\% of patients who underwent PAE reported experiencing complications at 12 months. Of the patients who underwent TURP, $84 \%$ of patients reported complications at 12 months.

A summary of expected QALY and cost values and corresponding ICER values for the cost-utility analysis and sensitivity analyses can be found in supporting information (online supplemental table S5).

\section{Sensitivity analysis}

Sensitivity analysis 1

Figure 1 shows an expected QALY value of 0.96 and 0.98 for PAE and TURP, respectively. The expected cost per patient was $£ 2009.50$ for PAE and £3067.14 for TURP. This generated an ICER of $£ 52445.86$ saved per QALY lost.

Sensitivity analysis 2

Figure 2 shows an expected QALY value of 0.94 and 0.91 for PAE and TURP, respectively. With an expected cost per patient remaining as $£ 2009.50$ for PAE and £3067.14 for TURP, the generated ICER was £32960.33 saved per QALY gained.

Sensitivity analysis 3

Sensitivity analysis 3 (seen in online supplemental figure S6) shows an expected QALY value of 0.94 and 0.91 for PAE and TURP, respectively. Taking into account the 


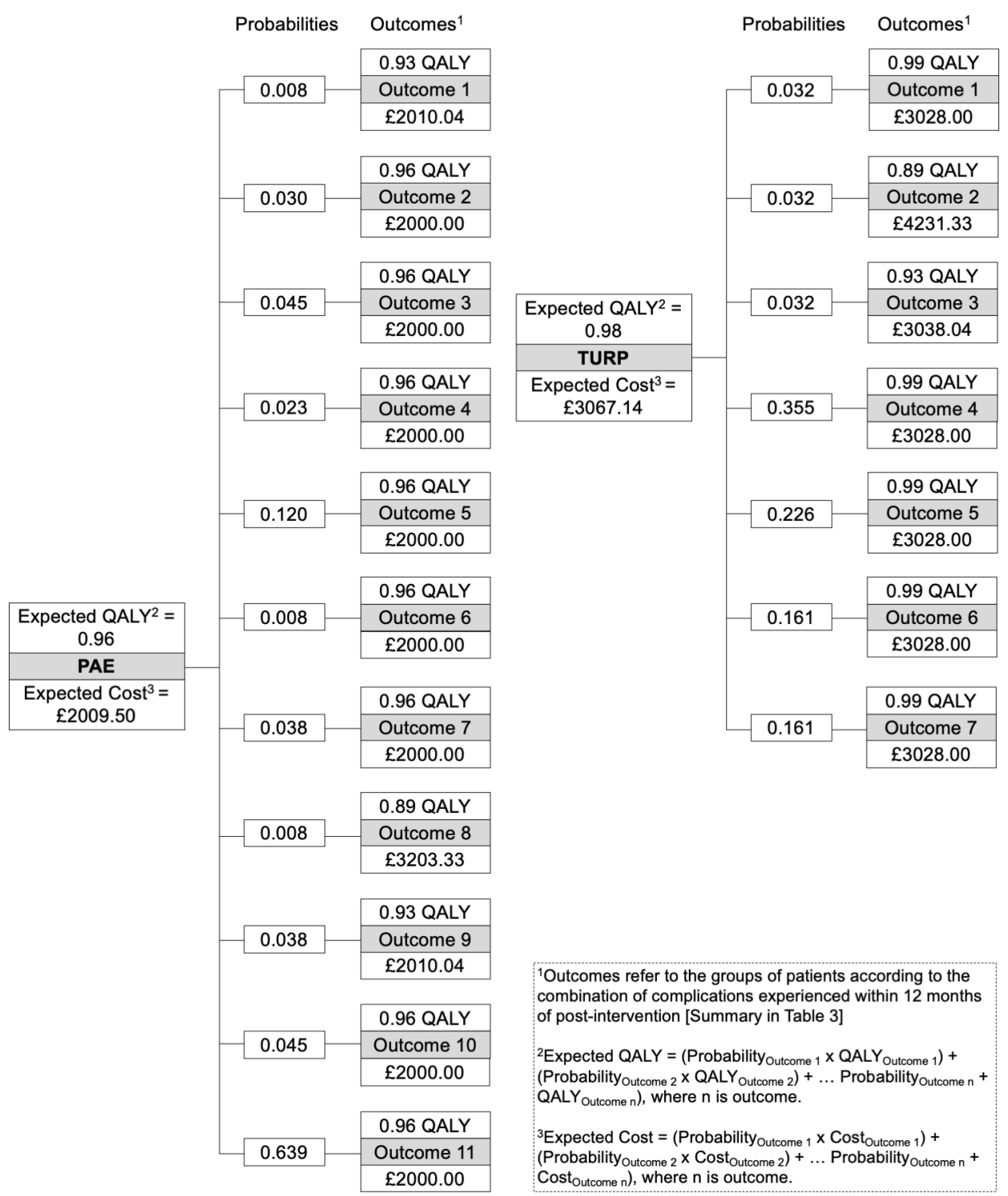

Figure 1 Decision tree showing sensitivity analysis 1 (including expected QALY and cost (in £ per patient). PAE, prostate artery embolization; QALY, quality-adjusted life year; TURP, transurethral resection of the prostate.

management of significant hematuria, the expected cost per patient was £2306.46 for PAE and £3958.96 for TURP. This generated an ICER of $£ 51498.80$ saved per QALY gained.

\section{DISCUSSION}

The main findings of this cost-utility analysis showed that PAE was more cost-effective compared with TURP at 1 year. PAE was associated with a lower average QALY value $(0.96 \pm 0.03)$ than TURP $(0.98 \pm 0.03)$ which suggests TURP remains a more clinically effective procedure. However, compared with TURP procedure costs (£3028), the costs incurred by PAE procedures ( $£ 2000$ ) were low enough to be cost-effective, as an ICER value of $£ 64798.10$ saved per QALY lost was greater than the upper NICE threshold of $£ 30000$ saved per QALY lost. ${ }^{25}$ This was demonstrated in a cost-effectiveness plane diagram (figure 3 ), where the
ICER sat in the left lower quadrant outside of the area bounded by the NICE thresholds.

This study is the first cost-utility analysis to use multicentre UK-based outcomes and costs, using information derived from the UK-ROPE Study. It is also the first of its kind to take into account the patient-reported complications that arise from PAE and TURP when determining which procedure is more cost-effective. Most PAE patients (85 out of 133) in the UK-ROPE Study did not experience the patient-reported complications considered in this cost-utility analysis 12 months post intervention, whereas most TURP patients did (26 out of 31 ).

Two sensitivity analyses were performed to account for the impact of patient-reported complications on QoL. When factoring in the cost of complications, the expected cost of PAE increased by $0.005 \%$ to $£ 2009.50$ per patient, while the expected cost of TURP increased further by $0.01 \%$ to $£ 3067.14$, thereby increasing the difference in 


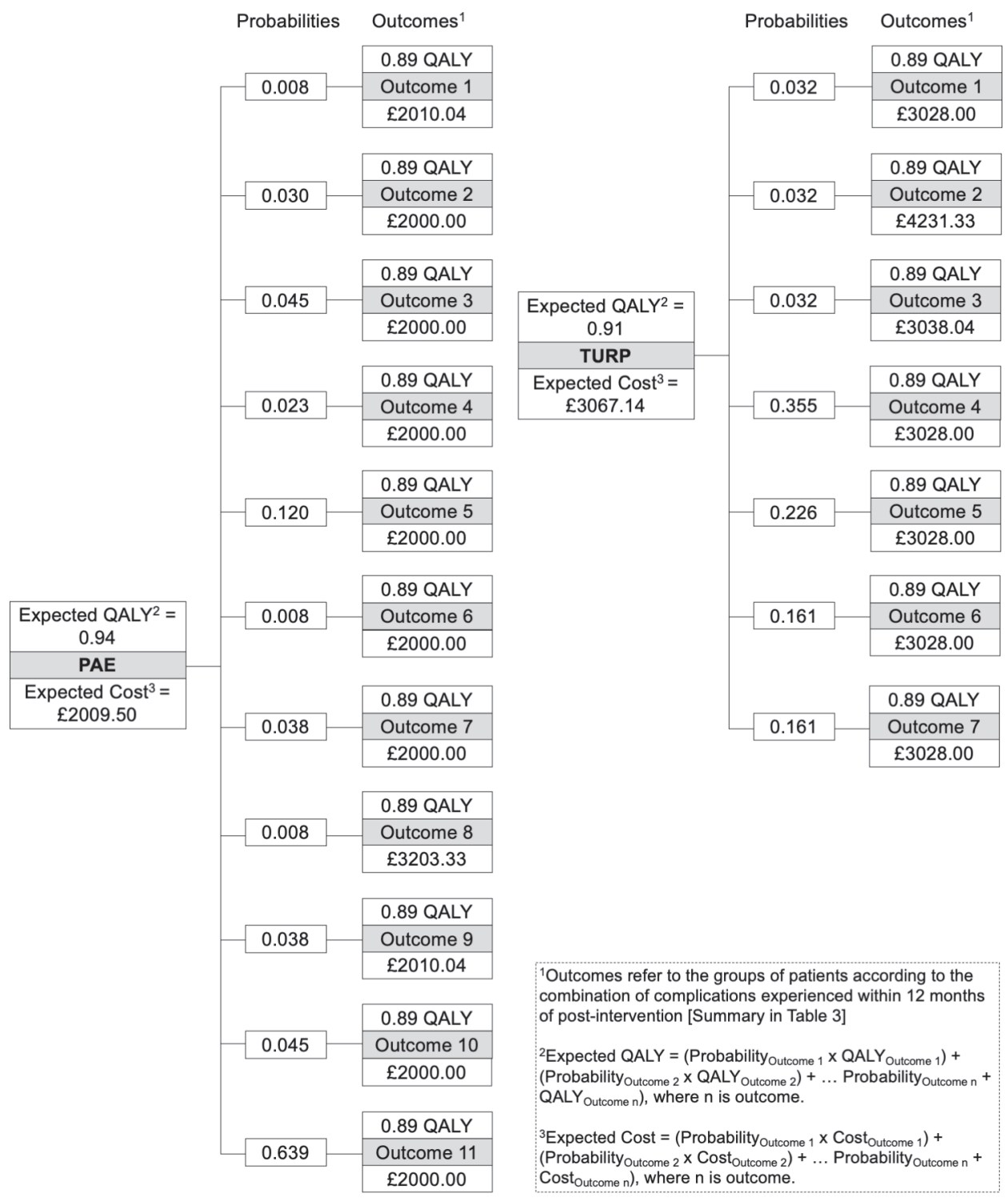

Figure 2 Decision tree showing sensitivity analysis 2 including expected QALY and cost (in £ per patient). PAE, prostate artery embolization; QALY, quality-adjusted life year; TURP, transurethral resection of the prostate.

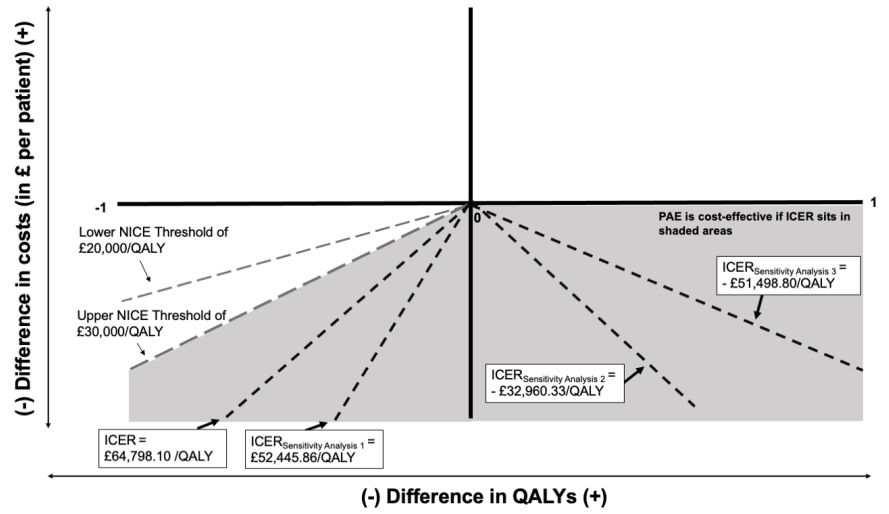

Figure 3 Cost-effectiveness plane diagram of PAE in comparison to the NICE cost-effectiveness threshold. ICER, incremental cost-effectiveness ratio; NICE, National Institute for Health and Care Excellence; PAE, prostate artery embolization; QALY, quality-adjusted life year. costs. These increases in expected cost are largely driven by the proportion of patients who experienced urinary infection and incontinence post intervention.

The first sensitivity analysis involved assigning all complications a QALY value based on the literature where available. This analysis showed although the ICER reduced to $£ 52445$.86 saved per QALY lost, PAE remained more costeffective than TURP when taking into consideration the impact of costs and QALY values associated with patientreported complications. The ICER remained outside of the area bounded by the NICE thresholds in the left lower quadrant of the cost-effectiveness plane diagram in figure 3 .

To further assess the impact of patient-reported complications on the cost-effectiveness, a secondary sensitivity analysis was conducted. This involved assigning all complications a QALY value of 0.89 , which represents the lowest QALY found in the literature among the complications. 
This generated an ICER of $£ 32960.33$ saved per QALY gained. Here, PAE remained cheaper than TURP when considering the impact of the cost of complications. PAE was also more effective than TURP when adjusting the QALYs associated with the complications to lower values; PAE was associated with an expected QALY value of 0.94 , while TURP was associated with an expected QALY value of 0.91 . The ICER sat in the right lower quadrant of the cost-effectiveness plane diagram in figure 3 , automatically making PAE more cost-effective than TURP.

The ICER values can be considered to be largely driven by the difference in costs of the two procedures. The PAE cost is largely driven by the cost savings associated with a shorter hospital stay and the lack of need for a traditional operating theatre. In addition to this, while this analysis took into account the costs of urodynamics, MRI and ultrasound scanning, these costs can be deemed optional. Removal of these costs would make the PAE cost cheaper and further increase the ICER, making PAE even more cost-effective. As it stands, the cost of PAE would need to increase by $35.5 \%$ ( $£ 710.71$ ) in order for the intervention to not be cost-effective over TURP. It is worth noting that at present, the availability of PAE across the UK is limited. Therefore, it is likely that there is potential for the costs of PAE to benefit from further economies of scale in the event that it becomes more widely commissioned.

As a retrospective analysis of a previously collected database which was not intended as part of the primary study, this study is limited by several related factors which should be carefully considered. A significant limitation of this analysis is the 1-year analytic horizon. Although we aimed to extend the analytic horizon to a longer follow-up duration, we were restricted by UK-ROPE data being available only up to 1 year. The QALY calculation requires the full IPSS breakdown being available (cost attributed to each individual symptom) and no dataset of follow-up greater than 1 year is available for this analysis. A limitation of this study was the lack of inclusion of certain procedure-related complications that occurred, which may have added to costs and reduced QoL. Early procedure-related (clinician reported) complications that were identified during the UK-ROPE Study included sepsis and groin hematoma, and while this analysis aimed to consider 1-year patient-reported complications, there is scope to evaluate the impact of doctor-related complications on cost-effectiveness of PAE. Future studies could increase the accuracy further through a randomised study to examine all forms of interventions for BPH. Additionally, the study was limited by centres being early in their PAE experience, due to the experimental nature of PAE at the commencement of the registry. PAE is known to have a learning curve (with more experienced operators having improved outcomes). However, this limitation would only serve to reduce the perceived costeffectiveness of PAE relative to TURP (which is a more established and commonly conducted intervention for $\mathrm{BPH})$ and therefore does not undermine the validity of this analysis. To mitigate the absence of a 'tariff' for PAE, procedural costs (for both PAE and TURP) were calculated from data at a single centre (UHS) in accordance with National Schedule of Reference Costs 2015/2016 ${ }^{15}$ published by the UK Department of Health and Social Care, but variability in these costs across other trusts will limit the reliability of the analysis.

\section{CONCLUSION}

While it is undeniable that the emergence of novel interventions to tackle BPH has benefited patients around the globe, the growing cost of medical expenditure has meant that these new technological advancements are posed with another dilemma, specifically the choice of treatment reliant on effective allocation of budget. This study adds to this debate, highlighting that PAE is not only cheaper than TURP in terms of direct costs but also it is more cost-effective when factoring in the potential complications and QoL improvement, during the first year only. Crucially however, it remains unclear over what time period PAE remains more cost-effective and investigating this further is essential, as it is entirely conceivable PAE could lose any superiority within the subsequent years of follow-up due to a higher reintervention rate.

Correction notice This article has been correctd since it was first published. The first author's orcid id has been inserted.

Twitter Sachin Modi @interradiologist

Contributors Planning of study: SM. Conception and design of study: SM, NCH, TJCB, NP and NY. Data acquisition and analysis: GV, NP, NY and LdP. Writing and review of manuscript: All authors. Guarantor of study: SM

Funding This work was independently conducted and did not receive any external financial support. The UK-ROPE Study was funded through multiple streams. The involvement of an independent academic evaluation centre 'Cedar' was funded through the National Institute for Health and Care Excellence. Procedure costs were covered either through local commissioning streams or through a grant from Cook Medical (Europe), where this was not possible.

Competing interests None declared.

Patient consent for publication Not applicable.

Ethics approval In the original ROPE study all procedures performed were in accordance with the ethical standards of the institutional and national research committee and with the 1964 Helsinki Declaration and its later amendments or comparable ethical standards. Specific ethical approval was not applicable for this study as it was a retrospective analysis of the original UK-ROPE data.

Provenance and peer review Not commissioned; externally peer reviewed.

Data availability statement All data relevant to the study are included in the article or uploaded as supplementary information.

Open access This is an open access article distributed in accordance with the Creative Commons Attribution Non Commercial (CC BY-NC 4.0) license, which permits others to distribute, remix, adapt, build upon this work noncommercially, and license their derivative works on different terms, provided the original work is properly cited, appropriate credit is given, any changes made indicated, and the use is non-commercial. See: http://creativecommons.org/ licenses/by-nc/4.0/.

\section{ORCID iDs}

Nikisha Patel http://orcid.org/0000-0002-1711-2151

Nathan Yung http://orcid.org/0000-0002-2745-8412

Bhaskar Somani http://orcid.org/0000-0002-6248-6478

Sachin Modi http://orcid.org/0000-0003-0813-3791 


\section{REFERENCES}

1 National Institute for Health and Care Excellence. Prostate artery embolisation for lower urinary tract symptoms caused by benign prostatic hyperplasia (Clinical guideline [IPG611]), 2018. Available: https://www.nice.org.uk/guidance/ipg611

2 Jung JH, McCutcheon KA, Borofsky M, et al. Prostatic arterial embolization for the treatment of lower urinary tract symptoms in men with benign prostatic hyperplasia. Cochrane Database Syst Rev 2020;12:CD012867.

3 Mayer EK, Kroeze SGC, Chopra S, et al. Examining the 'gold standard': a comparative critical analysis of three consecutive decades of monopolar transurethral resection of the prostate (TURP) outcomes. BJU Int 2012;110:1595-601.

4 Berry SJ, Coffey DS, Walsh PC, et al. The development of human benign prostatic hyperplasia with age. J Urol 1984;132:474-9.

5 Speakman M, Kirby R, Doyle S, et al. Burden of male lower urinary tract symptoms (LUTS) suggestive of benign prostatic hyperplasia (BPH) - focus on the UK. BJU Int 2015;115:508-19.

6 Bagla S, Smirniotopoulos J, Orlando J, et al. Cost analysis of prostate artery embolization (PAE) and transurethral resection of the prostate (TURP) in the treatment of benign prostatic hyperplasia. Cardiovasc Intervent Radiol 2017;40:1694-7.

7 Eredics K, Wachabauer D, Röthlin F, et al. Reoperation rates and mortality after transurethral and open prostatectomy in a long-term nationwide analysis: have we improved over a decade? Urology 2018;118:152-7.

8 Liao J, Zhang X, Chen M, et al. Transurethral resection of the prostate with preservation of the bladder neck decreases postoperative retrograde ejaculation. Wideochir Inne Tech Maloinwazyjne 2019;14:96-101.

9 Lourenco T, Armstrong N, N'Dow J, et al. Systematic review and economic modelling of effectiveness and cost utility of surgical treatments for men with benign prostatic enlargement. Health Technol Assess 2008;12:iii, ix-x, 1-146, 169-515.

10 Michalak J, Tzou D, Funk J. Holep: the gold standard for the surgical management of BPH in the 21(st) century. Am J Clin Exp Urol 2015;3:36-42.

11 Maclean D, Maher B, Modi S, et al. Prostate artery embolization: a new, minimally invasive treatment for lower urinary tract symptoms secondary to prostate enlargement. Ther Adv Urol 2017;9:209-16.

12 Abt D, Hechelhammer L, Müllhaupt G, et al. Comparison of prostatic artery embolisation (PAE) versus transurethral resection of the prostate (TURP) for benign prostatic hyperplasia: randomised, open label, non-inferiority trial. BMJ 2018;361:k2338.
13 Ray AF, Powell J, Speakman MJ, et al. Efficacy and safety of prostate artery embolization for benign prostatic hyperplasia: an observational study and propensity-matched comparison with transurethral resection of the prostate (the UK-rope study). BJU Int 2018;122:270-82.

14 Alcaraz A, Carballido-Rodríguez J, Unda-Urzaiz M, et al. Quality of life in patients with lower urinary tract symptoms associated with $\mathrm{BPH}$ : change over time in real-life practice according to treatment-the qualiprost study. Int Urol Nephrol 2016;48:645-56.

15 Department of Health and Social Care. Reference cost collection: national schedule of reference costs - year 2015-16 - NHS trust and nhs foundation trusts, 2016. Available: https://www.gov.uk/ government/publications/nhs-reference-costs-2015-to-2016

16 Kok ET, McDonnell J, Stolk EA, et al. The valuation of the International prostate symptom score (IPSS) for use in economic evaluations. Eur Urol 2002;42:491-7.

17 Olapade-Olaopa EO, Solomon LZ, Carter CJ, et al. Haematuria and clot retention after transurethral resection of the prostate: a pilot study. Br J Urol 1998;82:624-7.

18 National Institute for Health and Care Excellence (NICE). Scenario: management of a person with haematospermia, 2016. Available: https://cks.nice.org.uk/haematospermia

19 Monitor, NHS England. National tariff payment system 2015/16 (national prices), 2014. Available: http://www.gov.uk/government/ consultations/nhs-national-tariff-payment-system-201516engagement-documents

20 Verhamme KMC, Dieleman JP, Bleumink GS, et al. Incidence and prevalence of lower urinary tract symptoms suggestive of benign prostatic hyperplasia in primary care--the triumph project. Eur Urol 2002;42:323-8.

21 Jefferys A, Siassakos D, Wardle P. The management of retrograde ejaculation: a systematic review and update. Fertil Steril 2012;97:306-12.

22 National Institute for Health and Care Excellence. Preoperative tests (update) routine preoperative tests for elective surgery (NICE guideline NG45), 2016. Available: https://www.nice.org.uk/guidance/ ng45/resources/routine-preoperative-tests-for-elective-surgery1837454508997

23 NHS Prescription Service. 2015/2016 NHS prescription services drug tariff, 2014. Available: https://www.nhsbsa.nhs.uk/pharmacies-gppractices-and-appliance-contractors/drug-tariff

24 Armstrong $\mathrm{N}$, Vale L, Deverill $\mathrm{M}$, et al. Surgical treatments for men with benign prostatic enlargement: cost effectiveness study. BMJ 2009;338:b1288.

25 McCabe C, Claxton K, Culyer AJ. The NICE cost-effectiveness threshold. Pharmacoeconomics 2008;26:733-44. 